\title{
Solving Riccati differential equation using Fourier polynomial basis
}

\author{
Berna Bulbul Aslan \\ Department of Mathematics, Faculty of Science, Mugla University, Mugla, Turkey
}

Received: 8 January 2019, Accepted: 29 May 2019

Published online: 30 June 2019.

\begin{abstract}
A new matrix method based on polynomial approximation, using complex Fourier polynomial basis is presented for the solution of Riccati differential equations (RDE). The proposed method converts the governing differential equation of the system into a matrix equation, which corresponds to a system of nonlinear algebraic equations with unknown coefficients. The solution is calculated in the form of a series with easily computable components. Some numerical examples are included to demonstrate the validity and applicability of the technique.
\end{abstract}

Keywords: Riccati differential equations, matrix method, collocation points, Fourier polynomial basis.

\section{Introduction}

In this paper, a numerical method using Fourier polynomial basis is presented for the following RDE

$$
u^{\prime}(t)=p(t)+q(t) u(t)+r(t) u^{2}(t), 0 \leq t \leq T
$$

with the initial value

$$
u(0)=\alpha
$$

where $p(t), q(t)$ andr $(t)$ are given functions and $\alpha$ is an arbitrary constant, are a class of nonlinear differential equations of much importance, and play a significant role in many fields of applied science [1]. For instance, solitary wave solutions of a nonlinear partial differential equation can be expressed as a polynomial in two elementary functions satisfying a projective Riccati equation [2]. Such problems also arise in the optimal control literature. However, deriving an analytical solution in an explicit form seems to be unlikely to be achievable except for certain special situations [3]. Of course, if one knows a particular solution, then the general solution can be easily derived. For general cases, one must appeal to numerical techniques or approximate approaches for getting the solutions. Therefore, the problem has attracted much attention and has been studied by many authors (see e.g. [4-11] and the references cited therein).

In mathematics, a basis function is an element of a particular basis for a function space. Every continuous function in the function space can be represented as a linear combination of base functions. By using this property different polynomial approximation method using matrices studied by several researchers for the solution of ordinary and partial differential equations In Literature different polynomial basis used such as Taylor, Chebyshev, Legendre, Laguerre and Bessel series for matrix method [12-20]. But there is no paper used Fourier series basis.

It is obvious that Fourier series has significant advantage over the mentioned polynomials, Theorem 1 shows that the 
Fourier polynomial gives the best mean square approximation.

The method presented in this paper converts the given problem into a nonlinear algebraic system. By solving this system, the solution follows immediately. Our proposed method allows us to obtain an approximate solution of the given problem in a truncated Fourier series form. Our present approach avoids the tedious work needed by traditional techniques and existing numerical methods. It also avoids provides a solution with high accuracy, minimal calculations, and avoidance.

Theorem 1. If $\phi_{i}(x), i=1,2, \ldots$ form an orthonormal set of functions defined over $(a, b)$ then among all polynomials

$$
\phi_{n}(x)=\sum_{n=1}^{n} c_{m} \phi_{m}(x)
$$

of given degree $n$, that which gives the best mean square approximation to a function $f(x)$ defined in $(a, b)$ is the Fourier polynomial

$$
f_{n}(x)=\sum_{m=1}^{n}\left(f, \phi_{m}\right) \phi_{m}(x) .
$$

Proof. See ref. [21].

\section{Method of solution}

In mathematics a Trigonometric series is any series of the form:

$$
a_{0}+\sum_{n=1}^{\infty} a_{n} \cos n x+b_{n} \sin n x
$$

by means of the formula (3) the assumed solution $u(t)$ can be defined by the truncated Fourier polynomial as

$$
u_{2 N}(t)=\sum_{i=0}^{2 N} f_{i}(t) a_{i}
$$

Note that $a_{i}(i=0,1, \ldots, 2 N)$ are unknown coefficients which will be determined by the method. In general, the coefficients $a_{i}$ of the approximation are not equal to the Fourier coefficients; only if we obtain the exact solution of the problem will they be equal.

In the numerical solution of Eq. (1) with presented method, it is necessary to evaluate matrix form of (4).

$$
u_{2 N}(t)=\mathrm{F}(t) \mathrm{A}
$$

where

$$
\begin{aligned}
& \mathrm{F}(t)=\left[\begin{array}{llll}
f_{0}(t) & f_{1}(t) & \ldots & f_{2 N}(t)
\end{array}\right]_{1 \times(2 N+1)^{2}} ; \\
& f_{i}(t)=\left\{\begin{array}{l}
f_{0}(t)=1 \\
f_{2 i-1}(t)=\cos (i t) \\
f_{2 i}(t)=\sin (i t) \\
0 \quad \text { other }
\end{array}, i=1,2, \ldots, N, \mathrm{~A}=\left[\begin{array}{c}
a_{0} \\
a_{1} \\
\vdots \\
a_{2 N}
\end{array}\right] .\right.
\end{aligned}
$$

The relation between the matrix $u_{2 N}(t)$ and its derivative $u_{2 N}^{\prime}(t)$ is

$$
u_{2 N}^{\prime}(t)=\mathrm{F}^{\prime}(t) \mathrm{A}=\mathrm{F}(t) \mathrm{KA}
$$


where

$$
\mathrm{K}=\left[k_{i, j}\right]=\left\{\begin{array}{l}
k_{2 k, 2 k+1}=k \\
k_{2 k+1,2 k}=-k, k=0,1,2, \ldots, N ; i, j=0,1, \ldots, 2 N . \\
0 \quad \text { other }
\end{array}\right.
$$

By using the production of series, the matrix form of the $u^{2}(t)$ is obtained as

$$
u_{2 N}^{2}(t)=\tilde{\mathrm{F}}(t) \tilde{\mathrm{A}}
$$

where

$$
\begin{gathered}
\tilde{\mathrm{F}}(t)=\left[\begin{array}{lllll}
\tilde{\mathrm{F}}_{0}(t) & \tilde{\mathrm{F}}_{1}(t) & \ldots & \tilde{\mathrm{F}}_{2 N}(t)
\end{array}\right]_{1 \times(2 N+1)^{2}}, \\
\tilde{\mathrm{F}}_{i}(t)=\left[\begin{array}{llll}
f_{i}(t) f_{0}(t) & f_{i}(t) f_{1}(t) & \cdots & f_{i}(t) f_{2 N}(t)
\end{array}\right]
\end{gathered}
$$

and

$$
\tilde{\mathrm{A}}=\left[\begin{array}{llll}
\tilde{\mathrm{A}}_{0} & \tilde{\mathrm{A}}_{1} & \ldots & \tilde{\mathrm{A}}_{2 N}
\end{array}\right]_{(2 N+1)^{2} \times 1}^{T}, \tilde{\mathrm{A}}_{i}=\left[\begin{array}{llll}
a_{i} a_{0} & a_{i} a_{1} & \cdots & a_{i} a_{2 N}
\end{array}\right]^{T}, i=0,1, \ldots, 2 N .
$$

The method is based on computing the unknown coefficients by means of the collocation points. Two types of collocation points are used. Equally spaced points and Chebyshev-Gauss-Lobatto points (Unequally spaced points). A natural, and often convenient, choice for the collocation points is that of the equally spaced points given by $t_{s}=s h, h=\frac{T}{2 N}, s=$ $0,1, . ., 2 N,(? ?)$. Quite frequently, present method deliver more accurate solutions with the unequally spaced points which is defined as

$$
t_{s}=\frac{1}{2}\left(1-\cos \left(\frac{s}{2 N} \pi\right)\right) T, s=0,1,2, \ldots, 2 N
$$

for a domain $[0, T]$. To obtain numerical approximation to Eq. (1) under the given condition (2), we can reduce the Eq. (1) and (2) to the matrix equations as follows:

$$
\mathrm{F}(t) \mathrm{KA}=p(t)+q(t) \mathrm{F}(t) \mathrm{A}+r(t) \tilde{\mathrm{F}}(t) \tilde{\mathrm{A}}
$$

or briefly

$$
\mathrm{W}(t) \mathrm{A}-\mathrm{S}(t) \tilde{\mathrm{A}}=p(t) ;(\mathrm{W}(t)=\mathrm{F}(t) \mathrm{K}-q(t) \mathrm{F}(t), \mathrm{S}(t)=r(t) \tilde{\mathrm{F}}(t))
$$

By substituting the collocation points defined by (??) and (8) into Eq. (9) we have

$$
\mathrm{W}\left(t_{s}\right) \mathrm{A}-\mathrm{S}\left(t_{s}\right) \tilde{\mathrm{A}}=p\left(t_{s}\right), s=0,1,2, \ldots, 2 N
$$

or in the compact form

$$
\mathrm{WA}-\mathrm{S} \tilde{\mathrm{A}}=\mathrm{P}
$$

where

$$
\mathrm{W}=\left[\begin{array}{l}
\mathrm{W}\left(t_{0}\right) \\
\mathrm{W}\left(t_{1}\right) \\
\vdots \\
\mathrm{W}\left(t_{2 N}\right)
\end{array}\right], \mathrm{S}=\left[\begin{array}{l}
\mathrm{S}\left(t_{0}\right) \\
\mathrm{S}\left(t_{1}\right) \\
\vdots \\
\mathrm{S}\left(t_{2 N}\right)
\end{array}\right], \mathrm{P}=\left[\begin{array}{l}
p\left(t_{0}\right) \\
p\left(t_{1}\right) \\
\vdots \\
p\left(t_{2 N}\right)
\end{array}\right]
$$

Next, by means of relation (5) we can obtain the matrix form corresponding to the initial condition (2) as

$$
\mathrm{F}(0) \mathrm{A}=\alpha
$$


Table 1: Comparison of the absolute error in Example 1.

\begin{tabular}{|l|l|l|l|l|l|}
\hline Present method & Exact & $\begin{array}{l}\text { Equal } \\
\text { coll. } \\
(N=6)\end{array}$ & $\begin{array}{l}\text { Unequall } \\
\text { coll. } \\
(N=6)\end{array}$ & $\begin{array}{l}\text { Equall } \\
\text { coll. } \\
(N=9)\end{array}$ & $\begin{array}{l}\text { Unequall } \\
\text { coll. } \\
(N=9)\end{array}$ \\
\hline 0.1 & 0.110295196 & $0.436 \mathrm{e}-7$ & $0.301 \mathrm{e}-10$ & $0.385 \mathrm{e}-10$ & $0.541 \mathrm{e}-13$ \\
\hline 0.2 & 0.241976799 & $0.467 \mathrm{e}-7$ & $0.470 \mathrm{e}-9$ & $0.453 \mathrm{e}-10$ & $0.784 \mathrm{e}-13$ \\
\hline 0.3 & 0.395104848 & $0.543 \mathrm{e}-7$ & $0.509 \mathrm{e}-9$ & $0.892 \mathrm{e}-10$ & $0.124 \mathrm{e}-12$ \\
\hline 0.4 & 0.567812165 & $0.606 \mathrm{e}-7$ & $0.219 \mathrm{e}-8$ & $0.228 \mathrm{e}-10$ & $0.318 \mathrm{e}-12$ \\
\hline 05 & 0.756014392 & $0.663 \mathrm{e}-7$ & $0.329 \mathrm{e}-8$ & $0.135 \mathrm{e}-10$ & $0.423 \mathrm{e}-12$ \\
\hline 0.6 & 0.953566215 & $0.654 \mathrm{e}-7$ & $0.212 \mathrm{e}-8$ & $0.440 \mathrm{e}-10$ & $0.422 \mathrm{e}-12$ \\
\hline 0.7 & 1.152948966 & $0.626 \mathrm{e}-7$ & $0.424 \mathrm{e}-8$ & $0.123 \mathrm{e}-10$ & $0.210 \mathrm{e}-12$ \\
\hline 0.8 & 1.346363655 & $0.850 \mathrm{e}-7$ & $0.555 \mathrm{e}-9$ & $0.438 \mathrm{e}-10$ & $0.283 \mathrm{e}-12$ \\
\hline 0.9 & 1.526911312 & $0.998 \mathrm{e}-7$ & $0.814 \mathrm{e}-9$ & $0.548 \mathrm{e}-10$ & $0.267 \mathrm{e}-12$ \\
\hline 1 & 1.689498390 & $0.569 \mathrm{e}-7$ & $0.124 \mathrm{e}-10$ & $0.155 \mathrm{e}-10$ & $0.436 \mathrm{e}-12$ \\
\hline
\end{tabular}

To obtain the solution of Equation (1) under condition (2) fundamental solution matrix is formed as follows:

$$
\overline{\mathrm{W}} \mathrm{A}-\overline{\mathrm{S}} \tilde{\mathrm{A}}=\mathrm{P}
$$

where

$$
\overline{\mathrm{W}}=\left[\begin{array}{l}
\mathrm{W}\left(t_{0}\right) \\
\mathrm{W}\left(t_{1}\right) \\
\vdots \\
\mathrm{W}\left(t_{2 N}\right)
\end{array}\right], \overline{\mathrm{S}}=\left[\begin{array}{l}
\mathrm{S}\left(t_{0}\right) \\
\mathrm{S}\left(t_{1}\right) \\
\vdots \\
0
\end{array}\right], \mathrm{P}=\left[\begin{array}{l}
p\left(t_{0}\right) \\
p\left(t_{1}\right) \\
\vdots \\
\alpha
\end{array}\right]
$$

by solving (11), the unknown Fourier coefficients $a_{n}(n=0,1, \ldots, 2 N)$ are determined. Thus we get the solution.

\section{Numerical examples}

To give a clear overview of the content of this study, several RDEs will be tested with the above-mentioned method, which will ultimately show the efficiency and accuracy of this method. All the results here are computed using Maple v 14.01.

Example 1. As the first example, we consider a RDE as follows [6,7,9,11]:

$$
\left\{\begin{array}{l}
u^{\prime}(t)=1+2 u(t)-u^{2}(t), 0 \leq t \leq 4 \\
u(0)=0
\end{array}\right.
$$

the exact solution of this problem is

$$
u(t)=1+\sqrt{2} \tanh \left(\sqrt{2} t+\frac{\log \left(\frac{-1+\sqrt{2}}{1+\sqrt{2}}\right)}{2}\right) .
$$

The Fourier solution was obtained for $N=6$ and $N=9$. Numerical results are given in Table 1. The comparisons for equally spaced points and unequally spaced points, for present method have also been done. 
Table 2: The norms $\left\|u-u_{2 N+1}\right\|_{1},\left\|u-u_{2 N+1}\right\|_{2},\left\|u-u_{2 N+1}\right\|_{\infty}$ for $N=8,9,10$ in Example 2.

\begin{tabular}{|l|l|l|l|}
\hline$N$ & $\left\|u-u_{2 N+1}\right\|_{1}$ & $\left\|u-u_{2 N+1}\right\|_{2}$ & $\left\|u-u_{2 N+1}\right\|_{\infty}$ \\
\hline 8 & $0.45474784 \mathrm{e}-9$ & $0.14555323 \mathrm{e}-9$ & $0.57839634 \mathrm{e}-10$ \\
\hline 9 & $0.34538352 \mathrm{e}-10$ & $0.10997112 \mathrm{e}-10$ & $0.42005775 \mathrm{e}-11$ \\
\hline 10 & $0.32572292 \mathrm{e}-11$ & $0.82641502 \mathrm{e}-12$ & $0.37841498 \mathrm{e}-12$ \\
\hline
\end{tabular}

Example 2. Consider the equation (1) with

$$
\left\{\begin{array}{l}
p(t)=1 /(1+t), \\
q(t)=1 \\
r(t)=-1 \\
\alpha=1
\end{array}\right.
$$

The exact solution of this problem is

$$
u(t)=\frac{1}{1+t} .
$$
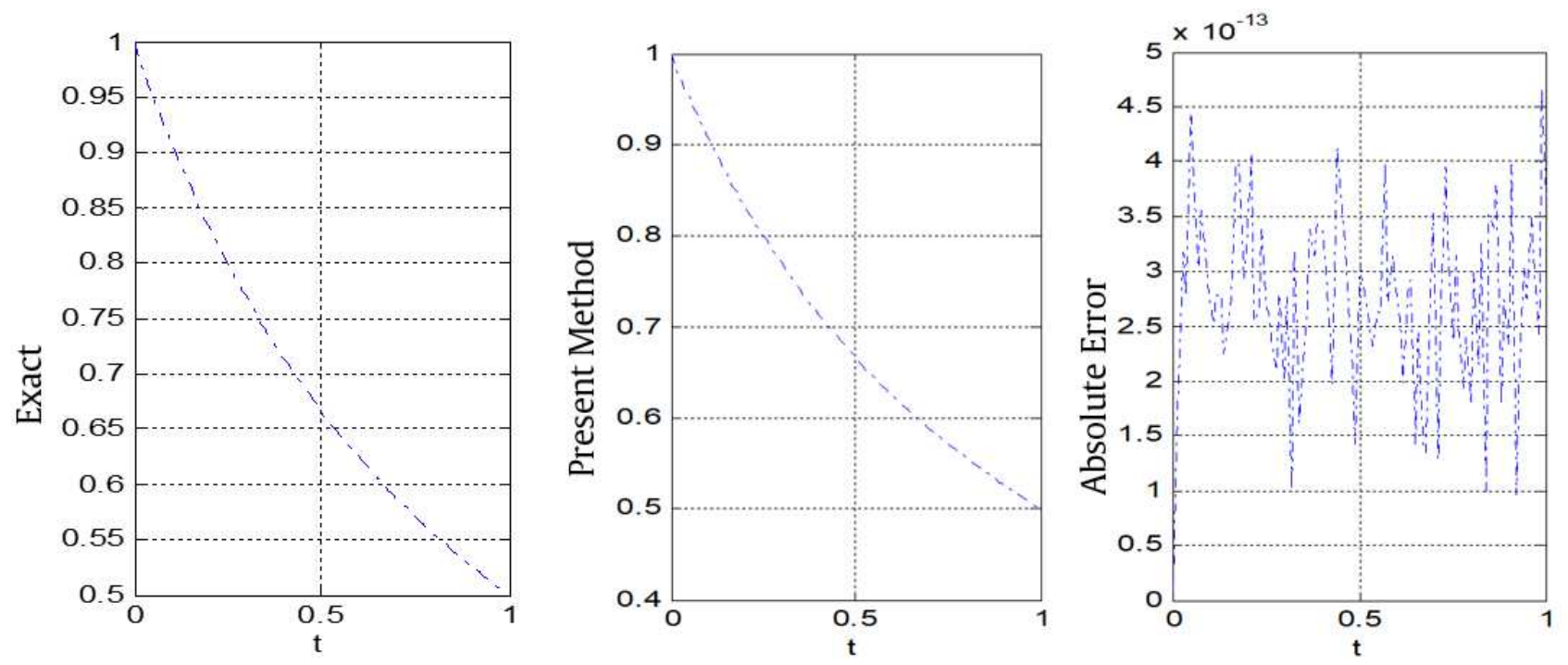

Fig. 1: Plot of the exact solution, the approximate solution and the error function in Example 2.

Table 2 represents different error norms obtained in solving Example 2. Figure 1 support the efficiency and the accuracy of the present method. From Fig. 1 we see that we can achieve a good approximation with the exact solution.

\section{Conclusions}

A matrix method has been presented for the RDEs. The applicability of the method is demonstrated by numerical examples which show good agreement with the exact solutions. The major advantage of the present method is that it is simple, straightforward and systematic when compared with other techniques. 


\section{Competing interests}

The authors declare that they have no competing interests.

\section{Authors' contributions}

All authors have contributed to all parts of the article. All authors read and approved the final manuscript.

\section{References}

[1] W.T. Reid, Riccati Differential Equations, Academic Press, New York, 1972.

[2] J.F. Carinena, G. Marmo, A.M. Perelomov, M.F. Ranada, Related operators and exact solutions of Schrödinger equations, Internat. J. Modern Phys. A 13 (1998) 4913-4929.

[3] M.R. Scott, Invariant Imbedding and its Applications to Ordinary Differential Equations, Addison-Wesley, 1973.

[4] Y. Tan, S. Abbasbandy, Homotopy analysis method for quadratic Riccati differential equation, Commun. Nonlinear Sci. Numer. Simul. 13 (2008) 539-546.

[5] B. Q. Tang, X. F. Li, A new method for determining the solution of Riccati differential equations, Appl. Math. Comput. 194 (2007) 431-440.

[6] S. Abbasbandy, Iterated He's homotopy perturbation method for quadratic Riccati differential equation, Appl. Math. Comput. 175 (2006) 581-589.

[7] M.A. El-Tawil, A.A. Bahnasawi, A. Abdel-Naby, Solving Riccati differential equation using Adomian's decomposition method, Appl. Math. Comput. 157 (2004) 503-514.

[8] H. Bulut D. J. Evans, On the solution of the Riccati equation by the decomposition method, Int. J. Comput. Math. 79 (2002) 103-109.

[9] S. Abbasbandy, Homotopy perturbation method for quadratic Riccati differential equation and comparison with Adomian's decomposition method, Appl. Math. Comput. 172 (2006) 485-490.

[10] S. Abbasbandy, A new application of He's variational iteration method for quadratic Riccati differential equation by using Adomian's polynomials, J. Comput. Appl. Math. 207 (2007) 59-63.

[11] F. Z. Geng, Y. Z. Lin, M.G. Cui, A piecewise variational iteration method for Riccati differential equations Comput. Math. Appl. 58 (2009) 2518-2522.

[12] B. Bülbül, M. Sezer, A Taylor matrix method for the solution of a two-dimensional linear hyperbolic equation , Appl. Math. Lett. 24 (2011) 1716-1720.

[13] B. Bülbül, M. Gülsu, M. Sezer, A new Taylor collocation method for nonlinear Fredholm-Volterra integro-differential equations, Numer. Meth. Part. D. E. 26 (2010) 1006-1020.

[14] B. Bülbül, M. Sezer, Taylor polynomial solution of hyperbolic type partial differential equations with constant coefficients, Int. J. Comput. Math. 88 (2011) 533-544.

[15] A. Akyüz, M. Sezer, Chebyshev polynomial solutions of systems of high-order linear differential equations with variable coefficients, 144 (2003) 237-247.

[16] A. Akyüz, M. Sezer, A Chebyshev collocation method for the solution of linear integro-differential equations, Int. J. Comput. Math. 72 (1999) 491-507.

[17] Ş. Yüzbaşı, N. Şahin, M. Sezer, A Bessel polynomial approach for solving linear neutral delay differential equations with variable coefficients, J. Adv. Res. Differ. Equ. 3 (2011) 81-101.

[18] M. Gülsu, B. Gürbüz, Y. Öztürk, M. Sezer, Laguerre polynomial approach for solving linear delay difference equations, Appl. Math. Comput. 217 (2011) 6765-6776.

[19] N. Akgönüllü, N. Sahin, M. Sezer, A Hermite Collocation Method for the Approximate Solutions of High-Order Linear Fredholm Integro-Differential Equations, Numer. Meth. Part. D. E. 27 (2011) 1707-1721.

[20] S. Yalçinbas, M. Sezer, H.H. Sorkun, Legendre polynomial solutions of high-order linear a Fredholm integro-differential equations, Appl. Math. Comput. 210 (2009) 334-349.

[21] I. N. Sneddon, Fourier series, Dover publication Inc, thirth edition, 1969. 